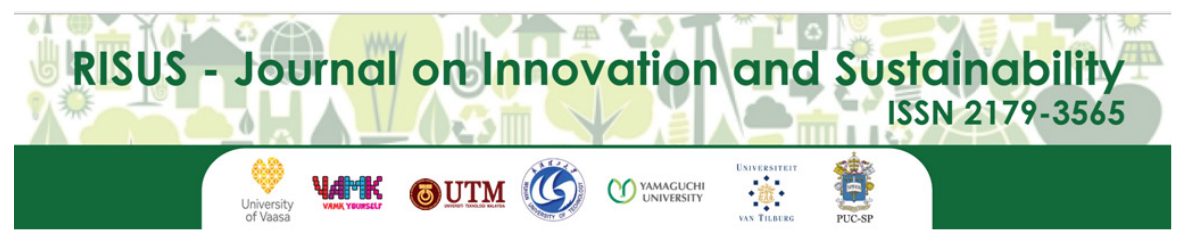

RISUS - Journal on Innovation and Sustainability volume 9, número 3 - 2018

ISSN: $2179-3565$

Editor Científico: Arnoldo José de Hoyos Guevara Editora Assistente: Lívia Lopes Aguiar Avaliação: Melhores práticas editoriais da ANPAD

\title{
MODELOS DE NEGÓCIOS APLICADOS A COMPARTILHAMENTO DE VEÍCULOS ELÉTRICOS
}

\author{
Business Models Applied to The Sharing of Electric Vehicles
}

\author{
Sarah Mesquita Lima, Vladia Celia Monteiro Pinheiro, José Dickson Araújo \\ de Oliveira, Carlos De Oliveira Caminha Neto, André Soares Lopes \\ Universidade De Fortaleza (UNIFOR), Fortaleza, Brasil \\ E-mail: sarahmesquitalima@gmail.com,vladiacelia@unifor.br, \\ dickson.araujo@unifor.br, carlos.o.c.neto@gmail.com,soareslopes@gmail.com
}

\begin{abstract}
It is very important that we may propose solution that allow us to diminish the consumption of gas and oil to run our light vehicle fleet. In this sense, a good alternative to the oil-dependent vehicles are the electric vehicles, although, consumers are resistant in adopting the electric car. A solution that was identified is electric car sharing. Considering the importance of promoting and diffusion of electric car-sharing, this paper aims to present the characterization of such business models implemented around the world, by using secondary-data analysis from each observed case, employing an adapted version of Weiller and Neely (2013) characterization model. The current research is a descriptive and qualitative based multiple-case study. The population is of 20 electric-car sharing models, located in 14 different countries. It was observed that the cost reduction of batteries accompanies the reduction of electric-car ownership cost. Moreover, the characterized business models did not make it any easier for technological innovation. It was also noted that predominantly, the risks and costs of electricity are borne by the service providers (taxed); that models encourage change in customer behavior; and that the models are not advantageous for long distances, given the low autonomy of the vehicles and restrictions of reach of the companies. Results corroborate Bohnsack, Pinkse and Kolk (2014), as it observes services-oriented business models. However, regarding the fear of limited vehicle autonomy, reported by Egbue and Long (2012) and Lim, Mak and Rong (2014), business models are not being elaborated and executed in the sense of solving it, which can be indicated as a discouraging factor to use of shared vehicles.
\end{abstract}

Key words:Business models; Electric vehicles; Sharing vehicles

\author{
Recebido em: 20/12/2017 \\ Aceito em: 01/09/2018
}




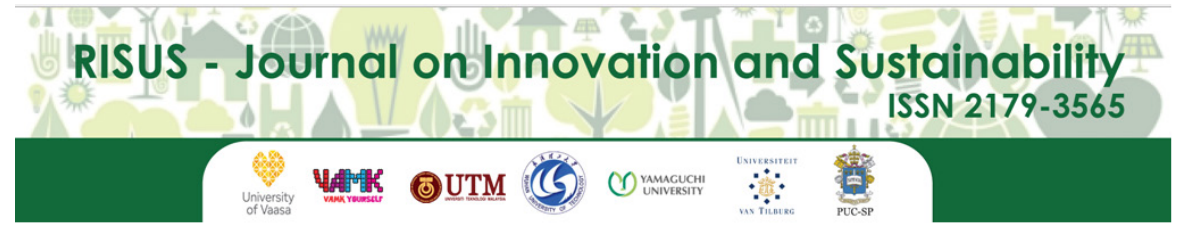

RISUS - Journal on Innovation and Sustainability volume 9, número 3 - 2018

ISSN: $2179-3565$

Editor Científico: Arnoldo José de Hoyos Guevara Editora Assistente: Lívia Lopes Aguiar Avaliação: Melhores práticas editoriais da ANPAD

\title{
MODELOS DE NEGÓCIOS APLICADOS A COMPARTILHAMENTO DE VEÍCULOS ELÉTRICOS
}

\author{
Business Models Applied to The Sharing of Electric Vehicles
}

\begin{abstract}
Sarah Mesquita Lima, Vladia Celia Monteiro Pinheiro, José Dickson Araújo de Oliveira, Carlos De Oliveira Caminha Neto, André Soares Lopes

Universidade De Fortaleza (UNIFOR), Fortaleza

E-mail: sarahmesquitalima@gmail.com, vladiacelia@unifor.br, dickson.araujo@unifor.br, carlos.o.c.neto@gmail.com, soareslopes@gmail.com
\end{abstract}

\begin{abstract}
Resumo: É de extrema importância propor soluções que viabilizem a diminuição do consumo de derivados de petróleo pela frota de veículos leves. Neste sentido, uma boa alternativa aos veículos que dependem dos combustíveis fósseis são os veículos elétricos, no entanto, os consumidores são resistentes a adoção dos veículos elétricos. Uma solução identificada é o compartilhamento de veículos elétricos. Considerando a importância da promoção e difusão de modelos de compartilhamento de veículos elétricos, esta pesquisa objetiva apresentar a caracterização dos modelos de negócios de compartilhamento de carros elétricos executados pelo mundo por meio da análise de dados secundários dos cases observados, utilizando o modelo de Weiller e Neely (2013) adaptado. A presente pesquisa, do tipo descritiva e de natureza qualitativa, caracteriza-se como um estudo de casos múltiplos. A população compreende 20 modelos de compartilhamento de veículos elétricos executados em 14 países. Constatou-se que a redução do custo da bateria é acompanhada da redução do custo de propriedade do veículo, além de que os modelos de negócio não viabilizam a inovação tecnológica. Constatou-se ainda que predominantemente os riscos e os custos de eletricidade ficam a cargo dos prestadores do serviço (embutidos nas taxas); que os modelos incentivam a mudança no comportamento do cliente; e que os modelos não são vantajosos para longas distâncias, dada a baixa autonomia dos veículos e restrições de alcance das empresas. Os resultados da pesquisa corroboram com Bohnsack, Pinkse e Kolk (2014) ao observar modelos de negócios voltados ao serviço. No entanto, quanto ao receio do alcance dos veículos relatado por Egbue e Long (2012) e Lim, Mak e Rong (2014), os modelos não estão sendo executados no sentido de atendêlo, o que pode ser indicado um fator desencorajador ao uso dos veículos compartilhados.
\end{abstract}

Palavras-chave: Modelos de negócios; Veículos elétricos; Compartilhamento de veículos.

\author{
Recebido em: 20/12/2017 \\ Aceito em: 01/09/2018
}




\section{INTRODUCTION}

Estudo publicado em 2013, pela International Energy Agency, assegurou que o setor de transportes é aprisionado aos sistemas de energias fósseis, uma vez que apresenta demanda agregada em petróleo equivalente a mais de 46 milhões de barris diariamente (International Energy Agency [IEA], 2013). Espera-se que a demanda pelo petróleo cresça em $26 \%$ até 2035, ultrapassando 58 milhões de barris por dia (IEA, 2013). De acordo com Barassa (2015), a dependência dos combustíveis se dá, primordialmente, em função do crescimento do transporte rodoviário. Neste sentido, cabe ressaltar o estudo publicado em 2014 pelo IEA, o qual sugere que a frota mundial de veículos leves possivelmente será duplicada entre os anos 2010 e 2035 (IEA, 2014).

Acerca dos veículos leves, é relevante destacar que a IEA (2014) atestou ainda que a demanda por energia do transporte rodoviário é feita em $40 \%$ por veículos leves, os quais representam metade da mobilidade mundial de passageiros. Diante do apresentado, é inquestionável a necessidade de soluções que viabilizem a diminuição do consumo de derivados de petróleo pela frota de veículos leves (Barassa, 2015). Deste modo, muitos países desenvolvidos estão adotando medidas que culminam em diminuir a dependência dos veículos aos combustíveis fósseis (Wills \& La Rovere, 2010).

De acordo com Ornellas (2013, p. 44),

...uma alternativa de fonte de energia limpa e renovável para o petróleo é a hidroeletricidade, solução que aproveita a força da água corrente sem reduzir sua quantidade nem gerar subprodutos tóxicos. Embora somente 33\% do potencial hidrelétrico tenha sido aproveitado, esse percentual evita a emissão de gases correspondente à queima de 4,4 milhões de barris de petróleo/dia

Deste modo, uma boa alternativa aos veículos que dependem dos combustíveis fósseis são os veículos elétricos, no entanto, de acordo com Egbue e Long (2012), os consumidores são resistentes a adoção destes tipos de veículos. Mais ainda, as principais preocupações dos consumidores em relação aos veículos elétricos são: (1) o custo; (2) a autonomia do veículo; e (3) a infraestrutura de recarga. Neste mesmo sentido, Lim, Mak e Rong (2014) acrescentam barreiras psicológicas: (1) a ansiedade da revenda, relativa à preocupação de que o valor dos veículos elétricos usados são se mantenham no futuro, tornando difícil revender os carros; e (2) a ansiedade de alcance, que se refere à preocupação de que a autonomia dos veículos elétricos não seja suficiente para atender às necessidades do condutor. Levando em consideração a resistência dos consumidores ao produto fora do padrão utilizado, muitos países estão adotando medidas com vista a viabilizar a difusão dos carros elétricos. Uma solução de mobilidade urbana e de aproximação dos consumidores com o produto a ser promovido identificada é o compartilhamento de veículos elétricos, uma vez que Ornellas (2013) afirma que o consumo colaborativo, decorrente do compartilhamento, no mundo dos negócios, é utilizado como forma de redução de custos.

Considerando a importância da promoção e difusão de modelos de compartilhamento de veículos elétricos, esta pesquisa se propõe a responder a seguinte questão: Como os modelos de negócios aplicados a compartilhamento de veículos elétricos estão sendo desenvolvidos pelo mundo? Para responder ao questionamento supracitado, este artigo objetiva apresentar a caracterização dos modelos de negócios de compartilhamento de carros elétricos executados pelo mundo por meio da análise de dados secundários dos cases observados e utilizando o modelo de Weiller e Neely (2013). Com este intuito, o presente trabalho organiza-se nesta seção introdutória, em uma segunda seção de referencial teórico, em uma terceira seção em que apresentamos os procedimentos metodológicos utilizados, uma seção quatro, de resultados e considerações finais na quinta e última seção.

\section{REFERENCIAL TEÓRICO}

O modelo de negócios é uma arquitetura organizacional e financeira de um negócio (Teece, 2010), deste modo, não é um modelo matemático ou uma equação financeira (Lafranque, 2015). Por modelo de negócios compreende-se a lógica de criar, entregar e capturar valor para a organização (Osterwalder \& Pigneur, 2010) a partir de uma plataforma que conecta os recursos, processos e fornecedores da empresa (Nielsen \& Lund, 2012). Em uma outra abordagem, acerca de contextos inovativos, Chesbrough (2006) salienta que não há nenhum valor inerente a uma tecnologia em si, 
afirmando que o valor é determinado pelo modelo de negócios usado por trazê-lo para um mercado. A mesma tecnologia feita para o mercado através de diferentes modelos de negócios gerará valores diferentes. Uma tecnologia inferior aplicada em um modelo de negócio adequados tenderá a trazer melhores resultados que tecnologias superiores comercializadas por meio de modelos de negócios inadequados (Chesbrough, 2006).

Acerca deste assunto, Stampfl (2015) considera ser importante implementar análises estruturadas para monitorar os ambientes interno e externo no qual os modelos de negócios estão inseridos, com vistas a fornecer respostas a questões como: (1) quais os modelos de negócios presentes no mercado?; (2) no que os fornecedores, clientes, competidores estão inovando?; (3) quais modelos de negócio em mercados próximos estão emergindo?; (4) quais as tendências que estão moldando o ambiente do modelo de negócio?

Cabe destacar Osterwalder e Pigneur (2010), os quais desenvolveram a ferramenta, Business Model Canvas (BMC), com nove dimensões que cobrem os três pilares conceituais da definição de modelo de negócios, que são: (1) criação de valor (parcerias-chave, atividades-chave e recursos-chave); (2) entrega de valor (canais, segmento de clientes e relacionamentos com o cliente); e (3) captura de valor (estrutura de custo e fontes de receitas). Sobre esta ferramenta, Bonazzi e Zilber (2014) salientam que ela pode ser considerada mais completa que os demais modelos de negócios já desenvolvidos (Hedman \& Kalling, 2003, Lecocq, Demil \& Warnier, 2006, Johnson, Christensen \& Kagermann, 2008), por abordar detalhadamente o relacionamento de todos os componentes organizacionais internos e externos, bem como por evidenciar como essas se relacionam para criar e capturar o valor proposto pela organização.

Especificamente acerca de veículos elétricos, urge destacar as direções norteadoras apontadas por Kley, Lerch e Dallinger (2011) com vistas a viabilizar penetração dos carros elétricos no mercado de modo competitivo, as quais estão destacadas no Quadro 1.

\section{Quadro 1 - Direcionadores de viabilização dos veículos elétricos}

\begin{tabular}{|l|l|}
\hline Direcionador & Especificação \\
\hline $\begin{array}{l}\text { Melhor utilização da } \\
\text { capacidade } \\
\text { veículo }\end{array}$ & $\begin{array}{l}\text { Compartilhar veículos, por meio de projetos de carsharing, frotas de veículos } \\
\text { profissionais ou transporte público, é uma maneira de diluir os elevados custos entre } \\
\text { um grande número de clientes. }\end{array}$ \\
\hline $\begin{array}{l}\text { Estender a utilização } \\
\text { do veículo }\end{array}$ & $\begin{array}{l}\text { Aumentar as aplicações dos veículos elétricos com a finalidade de melhorar a } \\
\text { efíciência econômica dos mesmos, como por exemplo o conceito de vehicle-to-grid, } \\
\text { no qual o veículo se toma fomecedor de energia para o sistema elétrico. }\end{array}$ \\
\hline Segundo uso & $\begin{array}{l}\text { Reutilizar os componentes que não podem ser mais aproveitados no veículo elétrico, } \\
\text { como, por exemplo, reaproveitamento da bateria, o que aumentaria o valor residual } \\
\text { desse componente. }\end{array}$ \\
\hline $\begin{array}{l}\text { Aumento da } \\
\text { aceitação }\end{array}$ & $\begin{array}{l}\text { Viabilizar a aceitação dos veículos elétricos a partir dos fatores que são considerados } \\
\text { os maiores obstáculos à disseminação dosmesmos, como, por exemplo, a autonomia. } \\
\text { No caso do exemplo citado, a criação de um serviço baseado em uma infraestrutura } \\
\text { de recarga orientada para o consumidor seria uma solução economicamente } \\
\text { interessante para o cliente. }\end{array}$ \\
\hline
\end{tabular}

Fonte: Elaborada com base em Kley et al. (2011) e La franque (2015).

Por meio da análise do Quadro 1, percebe-se que há espaço para adicionar valor ao veículo elétrico de forma de torná-lo atrativo no mercado. No entanto, conforme destaca Lafranque (2015, p. 53), "para resolver as diversas barreiras técnicas e econômicas enfrentadas pelo veículo elétrico o negócio da mobilidade elétrica se tornará mais complexo e fará evoluir as relações entre os diferentes agentes da indústria automobilística".

É de suma importância que os diversos atores que orbitam o tema da mobilidade elétrica se integrem e, neste sentido, Abdekafi, Mokhotin e Posselt (2013) aceitam que provedores de serviços relacionados a VEs devem se juntar à rede de mobilidade elétrica, em especial produtores e distribuidores de energia. Esses últimos, neste contexto, se tornam atores que podem gerar lucros adicionais com a nova tecnologia. Por serem atores novos para no mercado automobilístico e, por isso, precisam de modelos de negócios inovadores (Abdekafi et al., 2013). Neste sentido, urge destacar a pesquisa de 
Avans (2017), a qual desenvolveu um framework integrativo para selecionar e avaliar os parceiros de co-inovação, que consiste em quatro "ajustes" entre os parceiros da aliança: um de estratégia, outro de operação, outro de relacionamento e um de ajuste de rede. Referido framework pode ser aplicado, com as devidas adaptações, no desenho das parcerias necessárias a viabilização de modelos de negócios.

Acerca de modelos de negócios aplicados a veículos, importa salientar ainda a pesquisa de Wells (2013), a qual salienta que o modelo de negócios prevalecente durante o século XX focava no veículo como um bem a ser vendido ao melhor custo possível, com pouca ênfase sobre os impactos deste após a venda. Desta forma, o foco do modelo de negócios tradicional aplicado a veículos foca o produto em si (veículos) e não os serviços de mobilidade. Adicionalmente ao tema, cabe chamar atenção para a pesquisa de Kley et al. (2011), a qual corrobora esta ideia, ao considerar que os modelos variam entre dois extremos: modelos dedicados aos produtos e os dedicados ao serviço.

Considerando este caminho evolutivo da elaboração de planos de negócios, salienta-se que tais modelos, quando aplicados a veículos elétricos, devem ser orientados prioritariamente ao serviço, pois, de acordo com Bohnsack, Pinkse e Kolk (2014), os modelos de negócios orientados para o produto não devem ser aplicados aos veículos elétricos, uma vez que suas características técnicas tornam o modelo de negócios tradicional inviável. Egbue e Long (2012) acreditam que sem uma abordagem inovadora ao modelo de negócios o veículo elétrico é somente uma alternativa mais cara e com menor autonomia que a do carro tradicional. Com isso, Lafranque $(2015$, p. 52) considera que "com a mera aplicação do modelo de negócios tradicional, o veículo elétrico não terá nenhuma chance de vencer no mercado", salientando, por fim, que é imprescindível focar no serviço de mobilidade viabilizar a competitividade veículo elétrico (Lafranque, 2015). No entanto, Kempton e Tómic (2005a) e Kempton e Tómic (2005b) evidenciam um modelo de negócio orientado ao produto que, segundo os autores, é viável em termos de aplicação. Em referido modelo de negócio, os veículos elétricos - V2G - são utilizados como um sistema de armazenamento de eletricidade que pode ser utilizado de forma automática pelo sistema nacional de distribuição de energia.

Conforme pôde ser observado, não existe unanimidade quanto a que modelos de negócios devese adotar. Neste sentido Abdekafi et al. (2013) salientam que os modelos empresariais utilizados como inspiração devem ser examinados quanto à sua adequação no novo contexto. Levando em consideração o supracitado, foram mapeadas pesquisas que objetivaram avaliar modelos de negócios no âmbito da mobilidade elétrica, são eles: Fernandes (2012), Weiller e Neely (2013) e Weiller, Shang, Neely e Shi (2015). Fernandes (2012) se propôs a definir um modelo estruturado e genérico, baseado em métodos morfológicos, com a finalidade de desenhar, descrever, caracterizar e analisar modelos de negócio no âmbito da mobilidade elétrica, sob uma perspectiva holística. Por sua vez, Weiller e Neely (2013) identificaram objetivamente 11 os critérios de avaliação dos modelos de negócios com fundamento em casos analisados e na literatura sobre energia elétrica aplicada a veículos. Por fim, Weiller et al. (2015), utilizando a metodologia de desenvolvida por Weiller e Neely (2013) analisaram quatro modelos de negócios inovadores desenvolvidos em três países para apoiar a comercialização de veículos elétricos.

\section{METODOLOGIA}

A presente pesquisa, do tipo descritiva e de natureza qualitativa, caracteriza-se como um estudo de casos múltiplos (Yin, 2010), envolvendo mais de um caso a ser analisado. A população compreende 20 modelos de compartilhamento de veículos elétricos executados em 14 países. Vale salientar que a escolha dos países foi feita considerando pesquisa de Gissler, Raab, Tix e Merk (2016), a qual identificou os fatores econômicos, políticos e tecnológicos que influenciam o mercado, bem como mapeou os mercados por país de acordo com sua atratividade. Considerando a disponibilidade de informações e Gissler et al. (2016), esta pesquisa irá mapear as características dos modelos de negócios nos seguintes países: Alemanha, Brasil, Canadá, China, Coréia do Sul, Estados Unidos, França, Índia, Japão, Noruega, Reino Unido e Suécia, além de Espanha e Itália, que foram incluídos em função de apresentarem modelos bastante populares. Os modelos analisados podem ser observados no Quadro 2. 


\section{Quadro 2 - Modelos de negócios (MN) analisados por País.}

\begin{tabular}{|c|c|c|c|c|c|}
\hline País & MN & País & MN & País & $\mathrm{MN}$ \\
\hline Alemanha & DriveNow & \multirow[t]{2}{*}{ Brasil } & \multirow{2}{*}{$\begin{array}{l}\text { Vamo } \\
\text { Carro Leve }\end{array}$} & \multirow[t]{2}{*}{ França } & Autolib \\
\hline Canadá & Communauto & & & & Citiz Alpes-Loire \\
\hline Coreia do Sul & SoCar & \multirow[t]{2}{*}{ Chima } & GreenGo & \multirow[t]{2}{*}{ EUA } & Bluelndy \\
\hline Japầo & Choimobi Yokohama & & Eyueche & & $\mathrm{Car} 2 \mathrm{Go}$ \\
\hline Noruega & Th!Nk & \multirow[t]{2}{*}{ Espanha } & SpainCar & \multirow[t]{2}{*}{ Itália } & Sharengo \\
\hline Remo Unido & E-Car & & RecordGo & & e-Go Carsharing \\
\hline Suécia & Sunfleet & & & India & ZoomCar \\
\hline
\end{tabular}

Fonte: Elaborado pelos autores.

Para viabilizar a comparação entre as características dos modelos negócios analisados, estes serão caracterizados considerando os itens que compõem a cadeia de valor esquematizada por Weiller e Neely (2013), os quais identificaram os critérios de avaliação dos modelos de negócios com fundamento em casos analisados e na literatura sobre energia elétrica aplicada a veículos. A estrutura da pesquisa citada permite a comparação de modelos de negócios aplicados a veículos elétricos de acordo com os caracterizados, os quais, conforme destacado anteriormente, foram compilados a partir da literatura acadêmica acerca de adoção de tecnologia, inovação, política energética, bem como relatórios de indústria e consultoria. O quadro foi concebido para incluir os principais elementos que as empresas devem considerar ao inovar seu modelo de negócios em ecossistemas de veículos elétricos. Cada modelo de negócio deve ser descrito por meio dos 11 caracterizadores classificados em quatro quadrantes, de forma que cada um deles reflete o tipo de vantagem competitiva: orientada para o negócio ou orientada para o cliente e vantagens financeiras ou vantagens estratégicas, conforme evidenciado na Figura 1.

Figura 1 - Modelo de Weiller e Neely (2013)

\section{Financeiro}

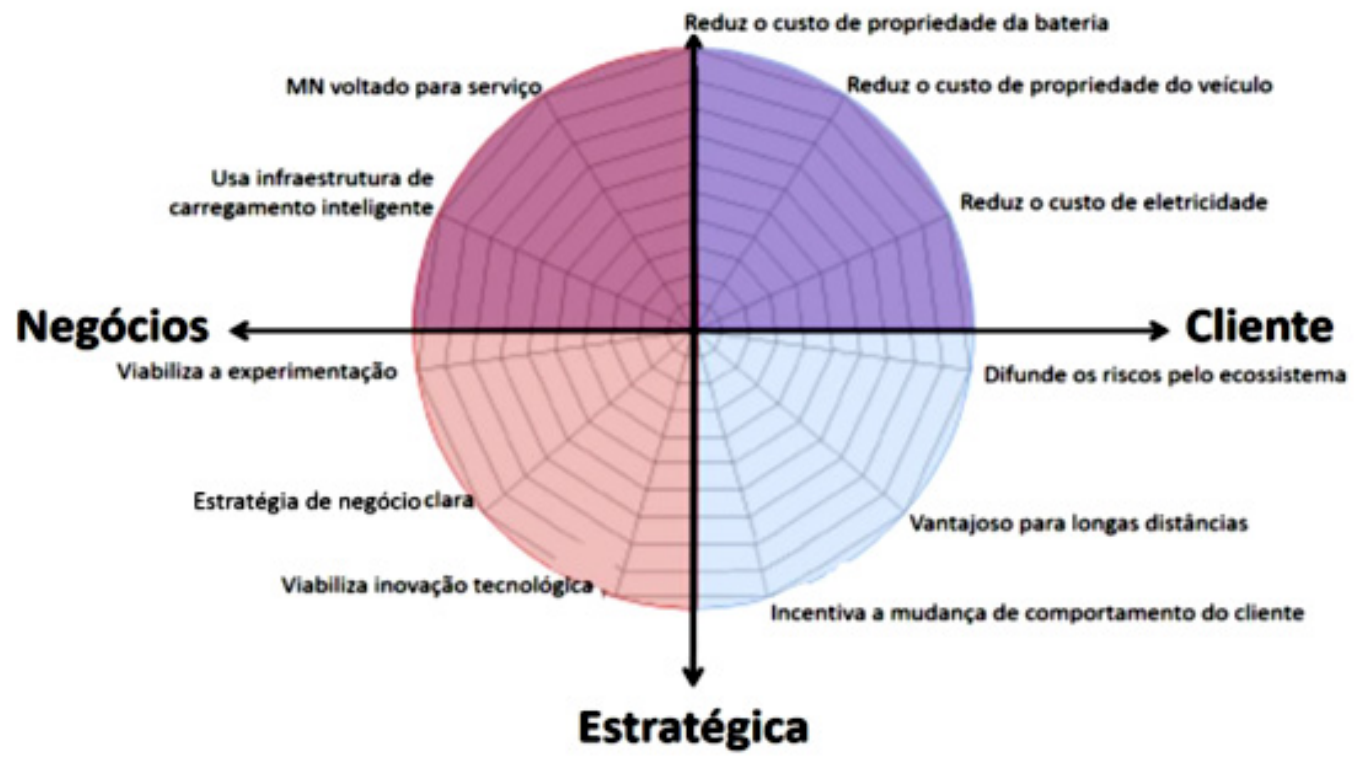

Fonte: Adaptado de Weiller e Neely (2013).

Os caracterizadores do quadrante "vantagens financeiras e voltadas ao cliente" evidenciados na Figura 1 avaliam como o modelo de negócios aborda considerações financeiras para os clientes. As três fontes de custo para os clientes são: bateria, o veículo (sem a bateria) e o preço da eletricidade como combustível (Andersen, Mathews \& Rask, 2009). Ainda sob a ótica dos clientes, analisa-se as principais barreiras para a adoção dos VEs pelos clientes que são: limitação dos VEs para viagens de longa distância, necessidade de mudança no comportamento do cliente e difusão dos riscos. Alguns modelos de negócios encontraram soluções para viagens de longa distância, enquanto outros estão limitados aos mercados locais. Há também uma escala de mudança no comportamento do consumidor de menos mudança de hábitos de condução do cliente para uma mudança significativa nas atitudes. Por 
fim, em relação à difusão dos riscos, escores mais altos indicam que os clientes assumem menos riscos que escores mais baixos (Weiller \& Neely, 2013).

Quanto às vantagens estratégicas do negócio, as características analisadas são a capacidade de moldar um ecossistema inovador (Geroski, 1990, Adner, 2006, Adner \& Kapoor, 2010), a formulação explícita ou implícita da inovação do modelo de negócios (Chesbrough, 2010, McNamara, Peck \& Sasson, 2011) e a abertura de inovação no modelo de negócios (Chesbrough, 2007).

Já em relação ao valor financeiro para o negócio, a cointegração das comunicações inteligentes entre os veículos e os sistemas de infraestrutura de energia elétrica é uma fonte de criação de valor complexo (Weiller \& Neely, 2013).

Por fim, ainda em relação ao valor financeiro para o negócio, este pode ser ampliado como resultado de modelos de negócios orientados para os serviços (Tukker, 2004). Neste sentido, importa salientar a importância dos serviços agregados como um diferencial competitivo a ser incorporado pelas empresas (Gianesi \& Correa, 1996).

Importa salientar que no modelo original, cada modelo de negócio pode ser classificado em escalas de 0 a 10, no entanto, nesta análise a escala será adaptada a variação de 0 a 2 . Referida adaptação se fez necessária, uma vez que no modelo original os caracterizadores eram pontuados por atores do sistema e esta pesquisa analisará os modelos de negócios com base nos dados secundários, sendo estes menos sensíveis quanto a percepção. A pontuação para cada caracterizador será alocada conforme evidenciado no Quadro 3.

Saliente-se ainda que, de acordo com Abdekafi et al. (2013), é importante capturar padrões nos modelos de negócios, uma vez que a partir desta captura é possível reduzir os diferentes tipos de modelos de negócios encontrados na prática para alguns padrões recorrentes. Deste modo, após a caracterização dos cases considerando o modelo Weiller e Neely (2013), com vistas a observar padrões nos modelos analisados, serão calculados coeficientes de correlação de Pearson, com vistas a observar associação entre os caracterizadores. Em relação ao coeficiente, será aplicado Pearson, técnica de estatística descritiva que mede o grau de dependência entre dois conjuntos de variáveis de escala métrica pareados e a direção dessa correlação - se positiva ou negativa (Mukaka, 2012). Referido coeficiente será operacionalizado com o uso do programa Microsoft Office Excel.

Quadro 3 - Especificação da pontuação por caracterizador.

\begin{tabular}{|c|c|c|c|}
\hline \multirow[b]{2}{*}{ Caracterizador } & \multicolumn{3}{|l|}{ Pontuacão } \\
\hline & 0 & 1 & 2 \\
\hline $\begin{array}{l}\text { Reduz o custo de } \\
\text { propriedadeda bateria }\end{array}$ & $\begin{array}{l}\text { Cliente financiou sem } \\
\text { nenhum subsidio a bateria. }\end{array}$ & $\begin{array}{l}\text { Cliente financiou com } \\
\text { subsidio a bateria }\end{array}$ & $\begin{array}{l}\text { Cliente não financiou a } \\
\text { bateria. }\end{array}$ \\
\hline $\begin{array}{l}\text { Reduz o custo de } \\
\text { propriedadedo } \\
\text { veículo }\end{array}$ & $\begin{array}{l}\text { Cliente financiou sem } \\
\text { nenhum subsidio o veiculo } \\
\text { (sem a bateria). }\end{array}$ & $\begin{array}{l}\text { Cliente financiou com } \\
\text { subsidio o veículo (sema } \\
\text { bateria). }\end{array}$ & $\begin{array}{l}\text { Cliente nao financiou o } \\
\text { veiculo (sema bateria). }\end{array}$ \\
\hline $\begin{array}{l}\text { Reduz o custo da } \\
\text { eletricidade }\end{array}$ & $\begin{array}{l}\text { Os custos derecarga sao os } \\
\text { preços de mercado e são } \\
\text { financiados pelo cliente. }\end{array}$ & $\begin{array}{l}\text { Os custos derecarga sao } \\
\text { abaixo dos de mercado e } \\
\text { são financiados pelo } \\
\text { cliente. }\end{array}$ & $\begin{array}{l}\text { Os custos derecarga estao } \\
\text { embutidos em taxas ou } \\
\text { financiados por terceiros. }\end{array}$ \\
\hline $\begin{array}{l}\text { Difunde os riscos pelo } \\
\text { ecossistema }\end{array}$ & $\begin{array}{l}\text { Os riscos de adoçao se } \\
\text { revertem prioritariamente } \\
\text { aos clientes. }\end{array}$ & $\begin{array}{l}\text { Os riscos sao distribuidos } \\
\text { por diferentes agentes. }\end{array}$ & $\begin{array}{l}\text { Os riscos de adoçao se } \\
\text { revertem a terceiros. }\end{array}$ \\
\hline $\begin{array}{l}\text { Vantajos o para longas } \\
\text { distâncias }\end{array}$ & $\begin{array}{l}\text { OMN nao res olve o } \\
\text { problema deautonomia para } \\
\text { longas distancias. }\end{array}$ & $\mathrm{NA}^{\mathrm{T}}$ & $\begin{array}{l}\text { OMN oferece soluçáo } \\
\text { para autonomia para } \\
\text { longas distâncias. }\end{array}$ \\
\hline $\begin{array}{l}\text { Incentiva a mudança } \\
\text { de comportamento do } \\
\text { cliente }\end{array}$ & $\begin{array}{l}\text { No promove alteracoes no } \\
\text { comportamento do cliente. }\end{array}$ & $\mathrm{NA}^{\mathrm{T}}$ & $\begin{array}{l}\text { Promove alteraços no } \\
\text { comportamento do cliente. }\end{array}$ \\
\hline $\begin{array}{l}\text { Wiabiliza a inovaçao } \\
\text { tecnológica }\end{array}$ & $\begin{array}{l}\text { OMN nao requer elou } \\
\text { promove mudanças } \\
\text { tecnológicas. }\end{array}$ & $\mathrm{NA}^{\mathrm{T}}$ & $\begin{array}{l}\text { OMN requer elou } \\
\text { promove a mudanças } \\
\text { tecnológica. }\end{array}$ \\
\hline $\begin{array}{l}\text { Estrategia de negocio } \\
\text { clara }\end{array}$ & $\begin{array}{l}\text { Estrategia inovadora, mas } \\
\text { seus componentes } \\
\text { inovadores não são } \\
\text { identificados explicitamente. }\end{array}$ & $\begin{array}{l}\text { Estrategia inovadora, seus } \\
\text { componentes inovadores } \\
\text { são identificados, mas não } \\
\text { detalhados explicitamente. }\end{array}$ & $\begin{array}{l}\text { Estrategia inovadora, seus } \\
\text { componentes inovadores } \\
\text { são identificados e } \\
\text { detalhados. }\end{array}$ \\
\hline $\begin{array}{l}\text { Viabiliza a } \\
\text { experimentação }\end{array}$ & $\begin{array}{l}\text { OMN requer açoes } \\
\text { irreversiveis. }\end{array}$ & $\begin{array}{l}\text { OMN pode apresentar } \\
\text { pequenas alteraçóes para } \\
\text { se adaptar às neces sidades } \\
\text { do mercado. }\end{array}$ & $\begin{array}{l}\text { OMN pode ser } \\
\text { substancialmente adaptado } \\
\text { às necessidades do } \\
\text { mercado }\end{array}$ \\
\hline $\begin{array}{l}\text { Usa infraes trutura de } \\
\text { carregamento } \\
\text { inteligente }\end{array}$ & $\begin{array}{l}\text { OMNutiliza uma } \\
\text { infraestrutura de } \\
\text { carregamento e cobrança } \\
\text { "muda". }\end{array}$ & $\mathrm{NA}^{\mathrm{T}}$ & $\begin{array}{l}\text { ONM utiliza uma } \\
\text { infraestrutura de } \\
\text { carregamento e cobrança } \\
\text { smart. }\end{array}$ \\
\hline $\begin{array}{l}\text { MVV voltado para } \\
\text { serviço }\end{array}$ & Veiculos como produto. & $\begin{array}{l}\text { Mobilidade como serviço } \\
\text { principal. }\end{array}$ & $\begin{array}{l}\text { Mobilidade como serviço } \\
\text { principale, } \\
\text { adicionalmente, serviços } \\
\text { agregados. }\end{array}$ \\
\hline
\end{tabular}




\section{RESULTADOS E DISCUSSÃO}

Considerando o objetivo da pesquisa, utilizando o modelo adaptado de Weiller e Neely (2013), foram desenvolvidos 20 gráficos do tipo radar, sendo um para cada modelo de negócio analisado. Com vistas a detectar padrões e possibilitar a redução dos diferentes tipos de modelos de negócios encontrados, os mesmos foram sobrepostos como forma de visualizar a predominância ou não de algum(ns) caracterizador(es), conforme Figura 2. Nesta figura, as linhas mais destacadas evidenciam os caracterizadores preponderantes, desta forma, observa-se que os modelos de negócios aplicados a compartilhamento de carro: (1) reduzem o custo de possuir a bateria; (2) reduzem o custo de possuir o veículo; (3) não viabilizam inovação tecnológica; (4) não utilizam infraestrutura de recarga inteligente; e (5) são voltados a prestação do serviço, sendo em $25 \%$ dos casos fornecidos serviços agregados.

\section{Figura 2 - Radar com a sobreposição dos MN de compartilhamento de carros analisados}

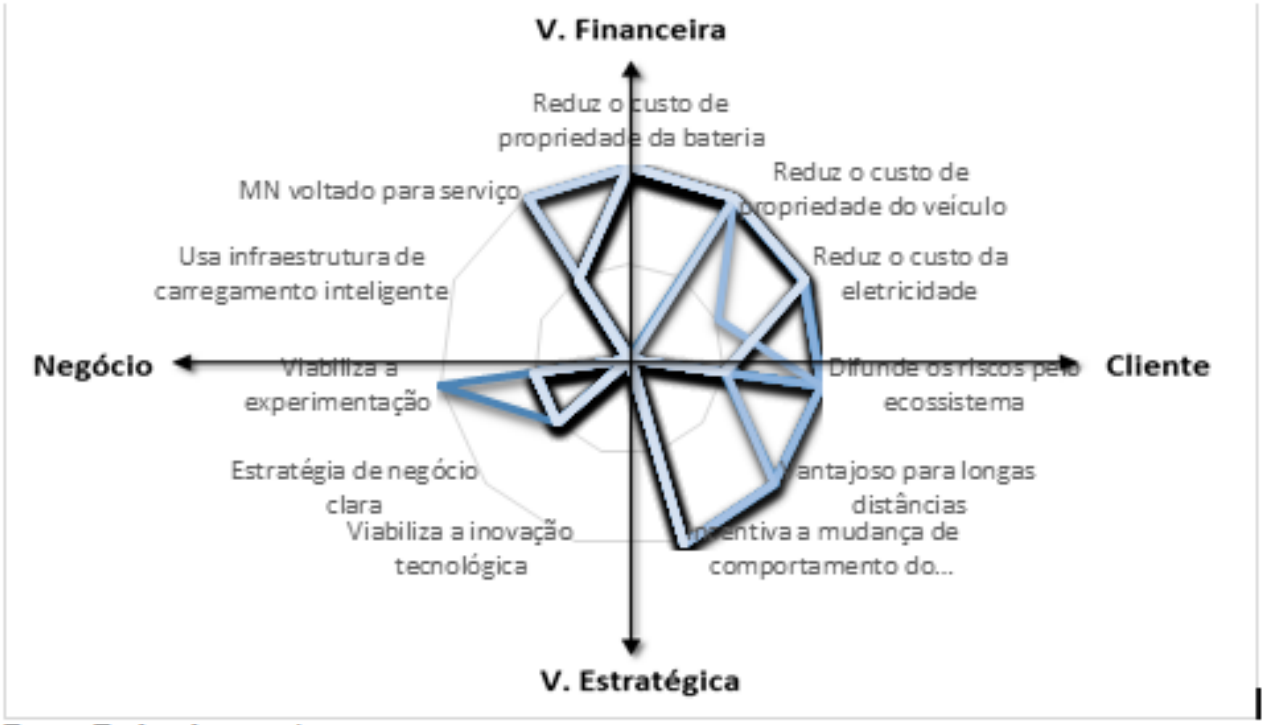

Fonte: Dados da pesquisa.

Considerando a distribuição normal dos caracterizadores, uma outra maneira de observar o comportamento dos caracterizadores é desenvolvendo um radar com as médias encontradas para os caracterizadores, conforme evidenciado na Figura 3.

\section{Figura 3 - Radar com a média dos MN de compartilhamento de carros analisados}

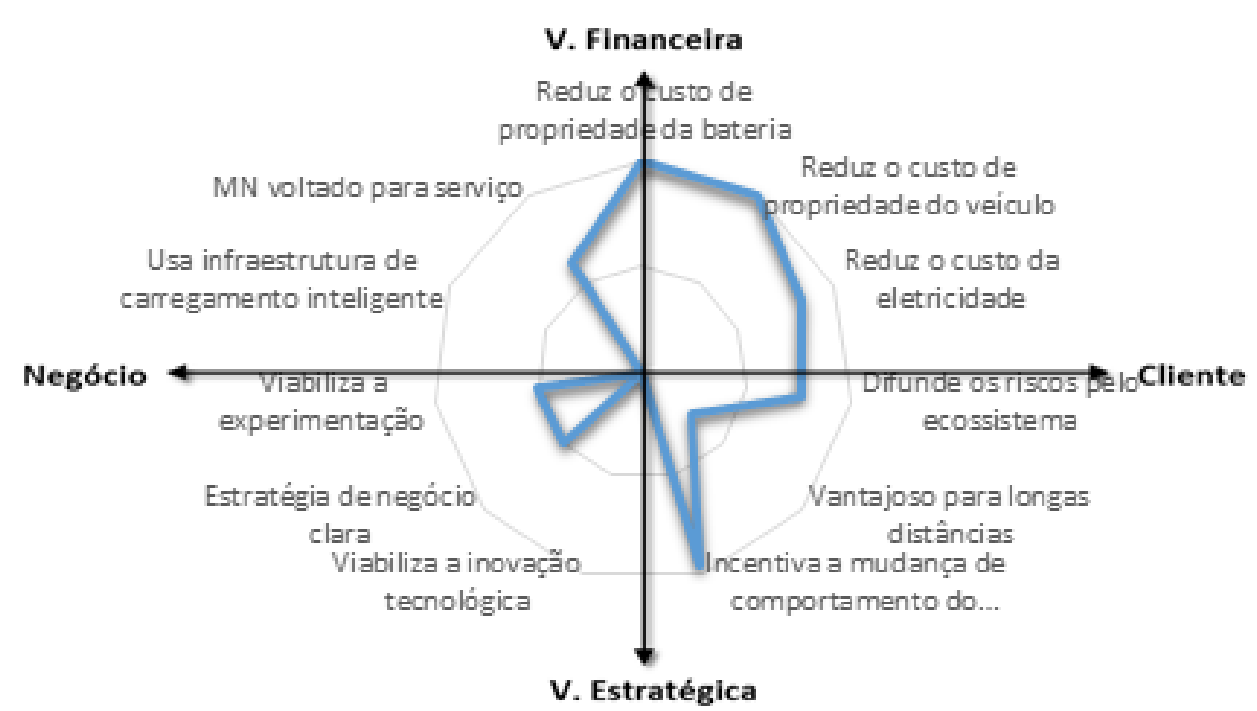

Fonte: Dados da pesquisa. 
Por meio da Figura 3 é possível inferir acerca da predominância dos modelos analisados, confirmando o já contatado na Figura 2 e trazendo com maior clareza que, predominantemente: (1) os riscos ficam a cargo dos prestadores do serviço (embutidos nas taxas); (2) os custos da eletricidade ficam a cargos dos prestadores do serviço (embutidos nas taxas); (3) incentivam a mudança no comportamento do cliente; e (4) os modelos não são vantajosos para longas distâncias.

Considerando os achados destacados a partir da observação das Figuras 2 e 3, importa salientar que os modelos de negócios que estão sendo executados pelo mundo atendem ao anseio identificado por Egbue e Long (2012) quanto ao custo para o cliente, uma vez que, em termos de quadrantes, percebe-se a predominância de modelos de negócios que enfatizam vantagens financeiras para os clientes. No entanto, quanto ao receio do alcance dos veículos relatado por Egbue e Long (2012) e Lim et al. (2014), os modelos não dirimem esta questão por uma questão de natureza tecnológica, considerando também que os mesmos não a viabilizam.

Ainda acerca dos radares das Figuras 2 e 3, importa destacar que foi observado que os modelos executados corroboram as ideias de Bohnsack et al. (2014) ao serem voltados ao serviço com a disponibilização ainda de serviços agregados, como a devolução dos carros de qualquer área de alcance do GPS e serviços de reserva online. Por fim, é válido observar a possível correlação entre os caracterizadores dos modelos analisados, a qual foi observada por meio do cálculo do coeficiente de Pearson, conforme Quadro 4.

\section{Quadro 4 - Coeficientes de correlação de Pearson}

\begin{tabular}{|c|c|c|c|c|c|c|c|c|c|c|}
\hline & RCB & RCV & RCE & $\overline{\text { DRE }}$ & VLD & IMC & VIT & ENC & VE & ICI \\
\hline RCB & & & & & & & & & & \\
\hline $\mathrm{RCV}$ & 00 & & & & & & & & & \\
\hline $\mathrm{RCE}$ & 0,192 & 0,192 & & & & & & & & \\
\hline DRE & 0,213 & 0,213 & 0,107 & & & & & & & \\
\hline VLD & $-0,141$ & $-0,141$ & $-0,104$ & 0,241 & & & & & & \\
\hline IMC & 0,411 & 0,411 & $-0,020$ & $-0,117$ & 0,194 & & & & & \\
\hline VIT & $-1,000$ & $-1,000$ & $-0,192$ & $-0,213$ & 0,141 & $-0,411$ & & & & \\
\hline ENC & $-1,000$ & $-1,000$ & $-0,192$ & $-0,213$ & 0,141 & $-0,411$ & 1,000 & & & \\
\hline $\mathrm{VE}$ & 0,000 & 0,000 & 0,000 & 0,309 & 0,342 & 0,000 & 0,000 & 0,000 & & \\
\hline ICI & $-1,000$ & $-1,000$ & $-0,192$ & $-0,213$ & $-0,141$ & $-0,411$ & 1,000 & 1,000 & 0,000 & \\
\hline MNS & 0,125 & 0,125 & $-0,170$ & 0,139 & 0,141 & $-0,308$ & $-0,125$ & $-0,125$ & 0,000 & $-0,125$ \\
\hline
\end{tabular}

Legenda: RCB - Reduz o custo de propriedade da bateria; RCV - Reduz o custo de propriedade do veículo; RCE - Reduz o custo da eletricidade; DRE - Difunde os riscos pelo ecossistema; VLD - Vantajoso para longas distâncias; IMC - Incentiva a mudança de comportamento do cliente; VIT - Viabiliza a inovação tecnológica; ENC - Estratégia de negócio clara; VE - Viabiliza a experimentação; ICI - Usa infraestrutura de carregamento inteligente; MNS - Modelo de negócio voltado para serviço.

Fonte: Dados da pesquisa.

Diante do Quadro 4 é possível inferir que nos modelos de negócios analisados, a redução do custo da bateria é acompanhada da redução do custo de propriedade do veículo, o que é perfeitamente adequado considerando que nos modelos de compartilhamento de veículo elétrico o automóvel é disponibilizado sempre com as baterias. Por fim, vale ressaltar ainda que foi observado que os modelos de negócio não viabilizam a inovação tecnológica, uma vez que não requer alterações nas tecnologias existentes para a operacionalização do modelo, o que também se aplica aos modos de recarga.

\section{CONSIDERAÇÕES FINAIS}

Este estudo objetivou apresentar a caracterização dos modelos de negócios de compartilhamento de carros elétricos executados pelo mundo como forma de observar como os modelos de negócios aplicados a compartilhamento de veículos elétricos estão sendo desenvolvidos pelo mundo. Por meio de uma pesquisa do tipo descritiva e de natureza qualitativa, foram analisados 20 modelos de compartilhamento de veículos elétricos executados em 14 países utilizando uma adaptação do modelo de Weiller e Neely (2013), além de uma análise por meio do coeficiente de Pearson.

Constatou-se que a redução do custo da bateria é acompanhada da redução do custo de propriedade do veículo, além de que os modelos de negócio não viabilizam a inovação tecnológica, uma vez que não requer alterações nas tecnologias existentes para a operacionalização do modelo, o que também se aplica aos modos de recarga. Além do supracitado, constatou-se que predominantemente os riscos e os custos de eletricidade ficam a cargo dos prestadores do serviço (embutidos nas taxas); que os modelos 
incentivam a mudança no comportamento do cliente; e que os modelos não são vantajosos para longas distâncias, dada a baixa autonomia dos veículos e restrições de alcance das empresas.

Os achados da pesquisa identificam que os modelos executados atendem aos anseios dos usuários de veículos elétricos quanto ao alto custo para o cliente (Egbue \& Long, 2012). A pesquisa corrobora ainda Bohnsack et al. (2014) ao observar modelos de negócios voltados ao serviço. No entanto, quanto ao receio do alcance dos veículos relatado por Egbue e Long (2012) e Lim et al. (2014), os modelos não estão sendo executados no sentido de atendê-lo, o que pode ser indicado um fator desencorajador ao uso dos veículos compartilhados.

Como limitação desta pesquisa, salienta-se a utilização de informações secundárias. O ideal seria a realização de entrevistas ou a aplicação de questionários aos gestores dos modelos de negócios analisados (Caminha, Furtado, Pinheiro \& Silva, 2016, Furtado et al. 2017, Lima \& Vasconcelos, 2016, Ponte, Caminha e Furtado, 2016).

Como limitação desta pesquisa, salienta-se a utilização de informações secundárias. O ideal seria a realização de entrevistas ou a aplicação de questionários aos gestores dos modelos de negócios analisados. Para pesquisas futuras, sugere-se a coleta de informações primárias, além da análise de modelos de negócios que utilizam veículos elétricos de outras maneiras, como transporte de funcionários, veículos de carga ou transporte púbico (ônibus e táxis elétricos), uma vez que por meio da utilização dos veículos elétricos em diversas áreas a difusão do mesmo se tornará mais efetiva. 


\section{REFERÊNCIAS}

Abdelkafi, N., Makhotin, S. \& Posselt, T. (2013). Business model innovations for electric mobilitywhat can be learned from existing business model patterns? International Journal of Innovation Management, 17 (1), 134-174.

Adner, R. \& Kapoor, R. (2010). Value creation in innovation ecosystems: How the structure of technological interdependence a effects firm performance in new technology generations. Strategic Management Journal, 31(3), 306-333.

Adner, R. (2006). Match your Innovation Strategy to your Innovation Ecosystem. Harvard Business Review, 98-107.

Andersen, P.H., Mathews, J. A. \& Rask, M. (2009). Integrating private transport into renewable energy policy: The strategy of creating intelligent recharging grids for electric vehicles. Energy Policy, 37(7), 2481-2486.

Avans, F. S. (2017). The Co-Innovation Alliance Scan, Tool for Effective Collaboration with External Partners. Journal on Innovation and Sustainability, 8(2), 170-179.

Barassa, E. (2015). Trajetória tecnológica do veículo elétrico: atores, políticas e esforços tecnológicos no Brasil. Dissertação (Mestrado em Política Científica e Tecnológica) - Instituto de Geociências, Universidade Estadual de Campinas, São Paulo.

Bohnsack, R., Pinkse, J. \& Kolk, A. (2014). Business models for sustainable technologies: Exploring business model evolution in the case of electric vehicles. Research Policy, 43 (2), 284-300.

Bonazzi, F. L. Z. \& Zilber, M. A. (2014). Inovação e Modelo de Negócio: um estudo de caso sobre a integração do Funil de Inovação e o Modelo Canvas. Revista Brasileira de Gestão de Negócios, 16(53), 616-637.

Caminha, C., Furtado, V., Pinheiro, V., \& Silva, C. (2016). Micro-interventions in urban transportation from pattern discovery on the flow of passengers and on the bus network. In Smart Cities Conference (ISC2), 2016 IEEE International, 1-6.

Chesbrough, H. W. (2006). Open innovation: The new imperative for creating and profiting from technology. Harvard Business Press.

Chesbrough, H. W. (2007). Have Open Business Models. MIT Sloan Management Review, 48(2), $22-28$.

Chesbrough, H. W. (2010). Business Model Innovation: Opportunities and Barriers. Long Range Planning, 43(2-3), 354-363.

Egbue, O. \& Long, S. (2012). Barriers to widespread adoption of electric vehicles: An analysis of consumer attitudes and perceptions. Energy Policy, 48, 717-729.

Fernandes, R. M. de F. (2012). Construção de um modelo de desenho/análise para modelos de negócio no âmbito da mobilidade elétrica: uma perspectiva holística. Dissertação (Mestrado em Gestão e Estratégia Industrial). Instituto Superior de Economia e Gestão. Universidade de Lisboa, Lisboa.

Furtado, V., Caminha, C., Furtado, E., Lopes, A., Dantas, V., Ponte, C., \& Cavalcante, S. (2017). Increasing the Likelihood of Finding Public Transport Riders that Face Problems Through a DataDriven approach. arXiv preprint arXiv:1705.03504. 
Geroski, P. A. (1990). Innovation, technological opportunity, and market structure. Oxford economic papers, 42(3), 586-602.

Gianesi, I. G. N. \& Correa, H. L. (1996). Administração Estratégica de serviços: operações para satisfação do cliente. São Paulo: Atlas.

Gissler, A., Raab, C., Tix, M., Merk, S. (2016). Electric Vehicle Market Attractiveness. Accenture, 2016. Recuperado em 30 março, 2017, de <https://www.accenture.com/t00010101T000000_w__/nzen/_acnmedia/PDF-37/accenture-electric-vehicle-market-attractiveness.pdf $>$.

Hedman, J. \& Kalling, T. (2003). The business model concept: theoretical underpinnings and empirical illustrations. European Journal of Information Systems, Basingstoke, 12(1), 49-59.

International Energy Agency [IEA]. (2013). Energy Policies of IEA Countries 2013 Review - Sweden, IEA, Paris.

International Energy Agency [IEA]. (2014). Energy technology perspectives 2014 - harnessing electricity's potential, IEA, Paris.

Johnson, M. W., Christensen, C. M. \& Kagermann, H. (2008). Reinventing your business model. Harvard Business Review, Boston, 86(9), p. 50-59.

Kempton, W. \& Tomić, J. (2005a). Vehicle-to-grid power fundamentals: Calculating capacity and net revenue. Journal of power sources, 144(1), 268-279.

Kempton, W. \& Tomić, J. (2005b). Vehicle-to-grid power implementation: From stabilizing the grid to supporting large-scale renewable energy. Journal of power sources, 144 (1), 280-294.

Kley F., Lerch C. \& Dallinger D. (2011). New business models for electric cars - A holistic approach. Energy Policy, 39, 3392-3403.

Lafranque, A. (2015). A emergência de modelos de negócios inovadores para apoiar o desenvolvimento da eletrificação veicular. Dissertação (Mestrado em Políticas Públicas, Estratégias e Desenvolvimento) - Instituto de Economia, Universidade Federal do Rio de Janeiro, Rio de Janeiro.

Lecocq, X., Demil, B. \& Warnier, V. (2006). Le business model, un outil d'analyse stratégique. L'Expansion Management Review, 4 (123), 96-109.

Lim, M. K, Mak, H. Y. \& Rong, Y. (2014). Toward Mass Adoption of Electric Vehicles: Impact of the Range and Resale Anxieties. Manufacturing \& Service Operations Management, 17 (1), 101-119.

Lima, S. M., \& de Vasconcelos, A. C. (2016). Ciclo de vida organizacional e ativos intangíveis de empresas de base tecnológica incubadas/Organizational life cycle and the structure of intangible assets of technology-based incubated companies. Revista de Administração de Roraima, 6(1), 128-155.

McNamara, P., Peck, S. I. \& Sasson, A. (2011). Competing Business Models, Value Creation and Appropriation in English Football. Long Range Planning.

Mukaka, M. M. (2012). A guide to appropriate use of correlation coefficient in medical research. Malawi Medical Journal, 24 (3), 69-71. 
Nielsen, C. \& Lund, M. (Eds.). (2012). Business model: networking, innovating and globalizing. Ventus Publishing Aps. Recuperado em 12 março, 2017, de <http://ebooksforexcellence.files.wordpress. com/2012/12/business-models.pdf $>$.

Ornellas, R. (2013). Impactos do consumo colaborativo de veículos elétricos na cidade de São Paulo. Future Studies Research Journal, 5 (1), 33-62.

Osterwalder, A. \& Pigneur, Y. (2010). Business model generation. New Jersey: John Wiley \& Sons.

Ponte, C., Caminha, C., \& Furtado, V. (2016). Busca Heurística de Melhor Caminho entre Dois Pontos quando Multiplas Origens e Multiplos Destinos São Possíveis. Anais do XIII Encontro Nacional de Inteligência Artificial e Computacional-ENIAC.

Stampfl, G. (2015). The Process of Business Model Innovation. Springer Gabler, forthcoming Summer.

Teece, D. J. (2010). Business models, business strategy and innovation. Long range planning, 43 (2), 172-194.

Tukker, A. (2004). Eight types of product-service system: eight ways to sustain-ability? Experiences from SusProNet. Business Strategy and the Environment, 13 (4), 246-260.

Weiller C. \& Neely A. (2013). Business model design in an ecosystem context. Cambridge Service Alliance, University of Cambridge.

Weiller, C., Shang, T., Neely, A. \& Shi, Y. (2015). Competing and co-existing business models for EV: lessons from international case studies. International Journal of Automotive Technology and Management, 15 (2), 126-148.

Wells, P. (2013). Sustainable business models and the automotive industry: A commentary, IIMB Management Review, 25 (4), 228-239.

Wills, W. \& La Rovere, E. L. (2010) Light vehicle energy efficiency programs and their impact on Brazilian CO 2 emissions. Energy Policy, 38 (11), 6453-6462.

Yin, R.K. (2010). Estudo de caso: planejamento e métodos / Robert K. Yin; Tradução Ana Thorell; revisão técnica Cláudio Damacena - 4. Ed. - Porto Alegre: Bookman. 